\title{
Politische Reformen als Auslöser großer Wählerwanderungen: Das Beispiel der Gemeindestrukturreform in der Steiermark 2013-2015
}

\author{
Florian Teurezbacher \\ IMC Fachhochschule Krems \\ Florian.Teurezbacher@fh-krems.ac.at
}

\section{Zusammenfassung}

Die Reformpartnerschaft aus SPÖ und ÖVP erlitt zwischen 2013 und 2015 in der Steiermark starke Verluste an den Wahlurnen. Vieles davon wurde als Folge der Gemeindestrukturreform dargestellt. Ziel dieses Beitrags ist es mittels einer Difference-inDifference-Schätzung den Effekt der aus der Gemeindestrukturreform resultierenden Fusionen zu berechnen. Die Ergebnisse der Regressionsanalysen weisen durchgängig auf den gleichen Trend hin: Ein leichter Malus für die ÖVP und ein leichter Bonus für die FPÖ in fusionierten Gemeinden (jeweils rund I-2 Prozentpunkte). Die SPÖ verliert durchwegs, während die kleinen Parteien (Grüne und KPÖ) nur marginal profitiert haben. Positive Auswirkungen gab es auch für Bürgerlisten, speziell dort wo der Protest am intensivsten war. Insgesamt zeigt sich aber, dass die Gemeindefusionen die starken Wählerwanderungen nur bedingt erklären.

\section{Schlüsselwörter}

Difference-in-Difference, Wahlergebnisse in der Steiermark, Gemeindefusionen, Reformen, Vetospieler

\section{Political Reforms as Trigger for a Serious Voter Shift: The Municipal Structural Reform in Styria 2013-2015}

\author{
Abstract \\ the voter shift.

\section{Keywords} \\ Difference-in-Difference, Electoral Outcomes in Styria, Municipal Reform, Veto-Player
}

The "Reformpartnerschaft" in Styria, consisting of the SPÖ and ÖVP, suffered severe losses in three elections from 2013 to 2015. A popular explanation pointed out that the municipal structural reform that has led to the merger of municipalities might have affected the election results. This article aims to find the real effect of the mergers by using a difference-in-differenceapproach. However, the patterns are very similar: A slight malus for the ÖVP and a small bonus for the Freedom Party in merged municipalities. The SPÖ lost throughout, while smaller parties (Greens and Communists) gained only marginally. Lists of citizens had big victories, where the resistance was strongest. Overall, the structural reform cannot explain the whole extent of

The author has declared that no competing interests exist. 


\section{Einleitung}

Die kommunale Struktur der Steiermark wurde mit OI.Jänner 2015 grundlegend verändert, statt $542 \mathrm{Ge}-$ meinden existieren nur noch 287. In Summe waren 385 Gemeinden von der steiermärkischen Gemeindestrukturreform betroffen und formten I30 neue Gemeinden, I57 Gemeinden blieben hingegen unverändert. ${ }^{I}$ Vor der finalen Umsetzung hatte es jahrelang breite Diskussionen gegeben. Am Höhepunkt der Proteste gingen 40 Beschwerden gegen einzelne Zusammenlegungen beim Verfassungsgerichtshof ein, die aber allesamt negativ beantwortet wurden. ${ }^{2}$ Auf der politischen Ebene kam es im Umfeld der Gemeindestrukturreform zu starken Umwälzungen, die sich bereits bei der Nationalratswahl 2013 äußerten, als die FPÖ knapp den ersten Platz in der Steiermark erreichte. Dieses Ergebnis stellt den Ausgangspunkt der folgenden Analyse dar und leitet auf die beiden regionalen Wahlen 2015 über, die Gemeinderatswahlen und Landtagswahlen. Diese waren unmittelbar von den Gemeindefusionen und anderen weniger populären Maßnahmen (wie Spitalsreform, Ausgabenkürzungen im Sozialbereich, Bezirksreform und Bezirksgerichtsschließungen) geprägt. Bereits die deskriptiven Statistiken weisen auf deutliche Verschiebungen hin (siehe Tabelle I).

Ziel dieses Artikels ist die Frage zu beantworten, wie stark die SPÖ-ÖVP-Landesregierung wegen der Gemeinstrukturreform von den Wählern „abgestraft“ wurde. Die theoretische Einordnung erfolgt anhand der Theorie zu Reformen sowie dem Vetospieler-Ansatz, die den Widerstand gegen Reformen und ihre Auswirkungen begründen. Wesentliche Aussage ist, dass sichtbare Einschnitte sichtbare Gewinner-Verlierer-Konstellationen erzeugen und dadurch den Wählerstimmenmarkt erschüttern (Mayntz/Streeck 2003). Es wird versucht eine detaillierte statistische Aufarbeitung der großen Wählerwanderungen zu bieten. Damit sollen die Wechselwirkungen zwischen Protest und Partizipation im skizzierten Spannungsfeld dargestellt werden. Der dazu verwendete Difference-in-Difference-Ansatz wird hauptsächlich in der Ökonomie eingesetzt, bietet sich aber auch bei manchen politikwissenschaftlichen Fragestellungen als Methode an (Garmann 20I7). Ziel ist es, den Effekt einer Maßnahme (im Vorher-Nachher-Vergleich zweier Gruppen) zu quantifizieren. Die wesentliche Annahme hierbei ist, dass ohne Eingriff die Wahlergebnisse in fusionierten und in nicht-fusionierten Gemeinden parallel verlaufen wären (Parallelitätsanahme).

I Das Steiermärkische Gemeindestrukturreformgesetz stellt alle Aspekte im Detail dar: https://www.ris.bka.gv.at/Dokumente/LgblAuth/LGBLA_ST_2OI4O4O2_3I/LGBLA_ST_2OI4O4O2_3I.pdfsig.

2 Der Verfassungsgerichtshof wies am 24.II.20I4 alle Anträge als unbegründet ab: https://www.vfgh.gv.at/downloads/ VfGH_G_6I-2OI4_Teufenbach.pdf.

\section{Theoretischer Rahmen und Literature Review}

\subsection{Reform-Theorie und Vetospieler-Ansatz}

Innerhalb demokratischer Systeme existieren Barrieren institutioneller, systemischer und politisch-gesellschaftlicher Art, die zu einer Verschleppung oder Verhinderung von Reformen führen können. ReformerInnen können in ihrem Tatendrang behindert werden, da es in vielen politischen Systemen eine Reihe von VetospielerInnen gibt, die dem entgegenstehen. Politische Stagnation ist wahrscheinlicher bei einer Vielzahl an VetospielerInnen, deren Inkongruenz sowie deren interner Kohäsion (Vgl. Tsebelis 1995, 293). Tsebelis definiert diese Starrheit als politische Stabilität: Diese stellt die Unmöglichkeit eines signifikanten Wandels des Status quo dar. Im Falle der Gemeindestrukturreform waren BürgermeisterInnen potentielle VetospielerInnen, da diese im System der Kommunalpolitik eine starke Rolle einnehmen (Welan 2000; Tsebelis 2000; Tsebelis 1999; Meyer 2012; Steininger 2004). VetospielerInnen waren im gegenständlichen Fall insbesondere in der Gemeindeinitiative zu finden, die als Reaktion auf die geplante Gemeindestrukturreform entstand.

In der Theorie werden Reformen anhand ihrer Intensität abgestuft. Reformen erster Ordnung seien laufende Anpassungen "gegebener Politikinstrumente an gegebene Ziele". Reformen zweiter Ordnung sind tiefgreifender, sie stellen Ergänzungen und einen Ersatz von Policies dar, „ohne die Ziele und die zugrunde liegende Philosophie der Politiksteuerung zu ändern". Reformen dritter Ordnung sind schließlich wirklich substantielle Eingriffe in die Strukturen, Ziele, Instrumente „und der zugrunde liegenden ,Philosophie“" (Schmidt 2005, 45). Die einzelnen reformpartnerschaftlichen Maßnahmen wurden in Tabelle 2 kategorisiert.

Die Theorie legt nahe, dass EntscheidungsträgerInnen das Risiko eher suchen, wenn sie sich in einer Verliererposition sehen und weniger, wenn sie sich auf der Siegerseite vermuten. Gleiches kann auf WählerInnen umgelegt werden: Wenn sich diese als VerliererInnen einschätzen, können Reformen einfacher umgesetzt werden. Auf der anderen Seite stehen WählerInnen Reformen skeptischer gegenüber, sobald sie etwas zu verlieren haben (Vis/van Kersbergen 2007, I68). Verlustängste wirken sich demnach stärker auf die Wahlentscheidung aus. Deshalb ist es nicht unrealistisch, dass Reformregierungen mit einem Regierungs-Malus statt eines Bonus zu rechnen haben (Acemoglu/Robinson 2000; Fernandez/Rodrik I99I; Buti, et al. 20I0). In der Steiermark beispielsweise war das Landesbudget speziell in der mittel- und langfristigen Prognose - ein wesentlicher Grund Reformen anzustrengen. Auffällig war, dass es eine teils starke Divergenz zwischen den unter PolitikerInnen populären Reformmaßnahmen und 
Tabelle 1: Zusammenfassung der deskriptiven Ergebnisse

\begin{tabular}{|c|c|c|c|c|c|c|c|c|c|c|c|c|}
\hline \multirow[b]{2}{*}{ Wahl } & \multicolumn{2}{|c|}{ SPÖ } & \multicolumn{2}{|c|}{ ÖVP } & \multicolumn{2}{|c|}{ FPÖ } & \multicolumn{2}{|c|}{ GRÜNE } & \multicolumn{2}{|c|}{ KPÖ } & \multicolumn{2}{|c|}{ SONSTIGE } \\
\hline & FUS & $\mathrm{NF}$ & FUS & $\mathrm{NF}$ & FUS & $\mathrm{NF}$ & FUS & $\mathrm{NF}$ & FUS & $\mathrm{NF}$ & FUS & NF \\
\hline NRW 2013 & $-5.92 \%$ & $-5.95 \%$ & $-5.32 \%$ & $-4.42 \%$ & $8.03 \%$ & $7.91 \%$ & $1.49 \%$ & $1.58 \%$ & $0.31 \%$ & $0.32 \%$ & & \\
\hline GRW 2015 & $-5.45 \%$ & $-4.67 \%$ & $-5.75 \%$ & $-1.77 \%$ & $8.32 \%$ & $5.09 \%$ & $1.65 \%$ & $0.62 \%$ & $0.40 \%$ & $0.25 \%$ & $1.26 \%$ & $0.38 \%$ \\
\hline LTW 2015 & $-10.59 \%$ & $-9.66 \%$ & $-10.20 \%$ & $-7.82 \%$ & $18.98 \%$ & $16.84 \%$ & $1.25 \%$ & $0.81 \%$ & $0.04 \%$ & $-0.34 \%$ & & \\
\hline
\end{tabular}

Tabelle 2: Kategorisierung der einzelnen Maßnahmen der Reformpartnerschaft

\begin{tabular}{|c|c|c|}
\hline Maßnahme & Ordnung & Begründung \\
\hline Verkleinerung des Landtages & 1 & seltene Maßnahme, widerspricht etwas der Ambition von mehr BürgerInnennähe \\
\hline Abschaffung Proporzregierung & 3 & $\begin{array}{l}\text { substantielle Änderung des politischen Entscheidungsfindungsmechanismus } \\
\text { durch klare Trennung in Opposition und Regierung }\end{array}$ \\
\hline Nulldefizit Landesbudget & $1-2$ & $\begin{array}{l}\text { könnte Paradigmenwechsel sein, wenn konsequent umgesetzt, da sämtliche } \\
\text { Leistungen des Landes in Frage gestellt werden könnten }\end{array}$ \\
\hline $\begin{array}{l}\text { Zusammenlegung von } \\
\text { Gemeinden }\end{array}$ & 2 & $\begin{array}{l}\text { Zeitpunkt und Art der Fusionen sprechen für höhere Bewertung, Fusionen } \\
\text { allerdings nur in Österreich als Steuerungsinstrument unpopulär }\end{array}$ \\
\hline $\begin{array}{l}\text { Zusammenlegung von } \\
\text { Bezirken }\end{array}$ & 2 & $\begin{array}{l}\text { staatlicher Aufbau nicht per se in Frage gestellt, Kostenreduktion als primäres } \\
\text { Anliegen }\end{array}$ \\
\hline $\begin{array}{l}\text { Abschaffung } \\
\text { Gratiskindergarten }\end{array}$ & 1 & $\begin{array}{l}\text { Kostenfrage wiederum zentral, obwohl Kürzung von Sozialausgaben auch } \\
\text { anders interpretiert werden könnte - als Verfolgung neoliberaler Ziele }\end{array}$ \\
\hline $\begin{array}{l}\text { Einsparungen bei } \\
\text { Sozialeinrichtungen }\end{array}$ & 1 & \\
\hline $\begin{array}{l}\text { Schließungen von Spitälern } \\
\text { und Spitalsabteilungen }\end{array}$ & 1 & \\
\hline
\end{tabular}

den von der Bevölkerung goutierten Teilaspekten gibt (Poier 20I6). Maßnahmen, die offensichtliche Verlierer erzeugten - wie etwa die Abschaffung des Gratiskindergartens - sind mit großer Mehrheit abgelehnt worden. Dabei wird die postulierte bessere Ausgabenkontrolle in den Augen der WählerInnen irrelevant. Die steirische Landesregierung hat das Risiko gesucht und hat die Auswirkungen tiefgreifender Reformen in ihre Agenda miteinkalkuliert. Ein Regierungs-Malus konnte in der folgenden Analyse auch für eine Einzelmaßnahme (die Gemeindestrukturreform) nachgewiesen werden.

\subsection{Bisherige Forschung}

Primärer Forschungsgegenstand in Bezug auf Gemeindefusionen ist die Wirkung auf die Ausgabendynamik der fusionierten Gemeinden. Die ausgewiesenen Effekte deuten in diversen Studien auf teilweise höhere, teilweise niedrigere oder gar keine signifikanten Ausgabenef- fekte hin. ${ }^{3}$ Einen guten Überblick bietet dazu die MetaStudie von Rösel (20I6b, 46). Skaleneffekte treten also nicht zwingend auf, sobald Gemeinden fusioniert werden. Deshalb sind die uneinheitlichen Ergebnisse nachvollziehbar. Effekte auf das Wahlverhalten sind hingegen nur in wenigen Studien analysiert worden. Gerade aber nicht-monetäre Kosten können stärker im politischen Gedächtnis der GemeindebürgerInnen verhaften. Diese sollen hier im Fokus stehen. Die Forschungslücke im Bereich der politischen Konsequenzen betrifft insbesondere die Auswirkungen auf die Wahlergebnisse, die kognitive Einschätzung von Gemeindefusionen wurde

3 Höhere Ausgaben werden beispielsweise von Fritz/Feld (2015; für Baden-Württemberg), Lüchinger/Stutzer (2002; Schweiz), Moisio/ Uusitalo (20I3; Finnland) oder Allers/Geertsema (20I6; Niederlande) nachgewiesen; niedrigere von Reingewertz (2012; für Israel) und Blom-Hansen et al. (20I4; Dänemark) nachgewiesen; Blesse/ Baskaran (20I6; für Brandenburg) können gar keine signifikanten Ausgabeneffekte eruieren. Im Falle von Kreisgebietsreformen in Deutschland findet Rösel (20I6a) ebenso keine signifikanten monetären Effekte. 
in einzelnen Studien recht gut untersucht. Für einen kurzen Überblick ist dazu Tavares (2018) zu empfehlen.

Dänische Studien kommen zum Schluss, dass die interne politische Wirksamkeit (Efficacy) der Gemeinden durch Fusionen stark beeinträchtigt wird. Hansen (2015) kann anhand von Befragungen eine signifikant gesunkene Zufriedenheit mit der Gemeindeverwaltung in Fusionsgemeinden feststellen. Die gleichen Schlussfolgerungen wurden auch schon von Lassen/Serritzlew (20IIa, 2OIrb) bei ähnlichen Studien gezogen. Die nichtmonetären Kosten - wie etwa weniger Demokratie auf Gemeindeebene und eine geringere politische Zufriedenheit der BürgerInnen - sind demzufolge substantiell. Die interne politische Wirksamkeit wird nachhaltig beeinträchtigt, die Demokratiequalität sinkt durch die geringere Bindung (bzw. größere Distanz) signifikant. Dadurch ist der Rückgang auch umso stärker, je stärker die Bevölkerungszahl durch die Fusionen zunimmt. Interessanterweise sinkt die Efficacy für alle Gruppen ${ }^{4}$ recht gleichmäßig, wodurch sich keine gestiegene Ungleichheit in der politischen Beteiligung (ein anderer Faktor der die demokratische Qualität wesentlich beeinflusst) ergibt. Auch in Japan führte ein unverhältnismäßig starker Rückgang öffentlicher Dienstleistungen in fusionierten Kleinst- und Kleingemeinden zu denselben Effekten. Dort wo aus der Mehrheit eine klare Minderheit wurde, erzeugte dies eine ungleiche Verteilung von Gewinnern und Verlierern - mit denselben negativen Folgen für den demokratischen Outcome (Yamada 2018). Diese Effekte sollten der Argumentation von Lassen und Serritzlew folgend als reale Kosten für die Demokratie aufgefasst werden.

\section{Politische Rahmenbedingungen}

In Folge einer leichten Wahlniederlage bei der Landtagswahl 2010 wurde die Reformpartnerschaft von Landeshauptmann Voves (SPÖ) und Landeshauptmannstellvertreter Schützenhöfer (ÖVP) paktiert. Die vorhergehende Legislaturperiode unter erstmaligem Vorsitz eines SPÖ-Landeshauptmannes war von beständigem Streit zwischen den beiden Großparteien geprägt. In der Steiermark verspürte man auf Grund der nicht allzu günstigen Zukunftsprognosen die Notwendigkeit zu bedeutenden Reformen und formte dazu eine auf zwei Perioden ausgelegte, überdimensionierte Koalition (Lehmbruch 1998). Die Eckpfeiler der Reformpartner-

4 In beiden Studien werden Difference-in-Difference-Schätzungen zur Internal Political Efficacy durchgeführt. In dem einem Fall ist der Einfluss der Bevölkerungsgröße der Fusionsgemeinde auf die Efficacy der zentrale Untersuchungsgegenstand, diese wird auf einer neunstufigen Skala um fast 2 Stufen schlechter eingestuft. In dem anderen Fall wird eine mögliche Zunahme der Ungleichheit der Efficacy nach Geschlecht und anhand von Altersgruppen differenziert getestet. schaft umfassten folgende Themenbereiche: Eine Demokratie-, eine Verwaltungs- sowie eine Spitals- und Gesundheitsreform. ${ }^{5}$ Die mediale Resonanz auf die Reformpartnerschaft war - wie Pekari (2016) nachweist - durchaus wohlwollend, wohl auch weil Medien die Reformen einforderten sonst selbst unglaubwürdig geworden wären. Die Ausgangslage für die Gemeindestrukturreform kann unter dem Eindruck von vier Problemfeldern zusammengefasst werden: Eine sehr kleinteilige Gemeindestruktur, eine problematische demografische Entwicklung, eine schwierige wirtschaftliche Situation der Gemeinden und ein eigeengter Spielraum durch den Stabilitätspakt (Vgl. Wlattnig 20I4, 248). ${ }^{6}$ Die 302 Gemeinden mit Bevölkerungsrückgang und jene 225 in Geldnöten waren erste Ansprechpartner. Die neue Struktur wurde in bis 3I.Jänner 2013 final festgelegt.

Bereits im September 2012 wurde eine Gemeindeinitiative durch BürgermeisterInnen formiert, mit dem Ziel die Gemeindestrukturreform zu verhindern oder zumindest abzuschwächen.7 Das Argumentationsmuster der Initiative fokussierte lange Zeit darauf, dass in IO3 Gemeinden Volksbefragungen durchgeführt wurden, von denen - unter teilweise hoher Partizipation - 89 negativ und I4 positiv ausgefallen waren. Von den ursprünglich I2I Gemeinden, die sich der Gemeindeinitiative angeschlossen hatten, wurden drei Viertel in die Gemeindestrukturreform einbezogen, 30 Gemeinden blieben eigenständig. Jene Gemeinden, die gegen eine Fusion vor dem Verfassungsgerichtshof geklagt haben, waren fast ausnahmslos in der Initiative vertreten gewesen. Gerade im Vorfeld der Nationalratswahl 2013 mobilisierte die Initiative gegen die Fusionen und plakatierte offen für ein „Abstrafen" der Reformpartner in der Wahlkabine. Vom Recht eine Volksabstimmung zu initiieren wurde dennoch nicht Gebrauch gemacht. ${ }^{8}$ Wlattnig (20I4) vermutet, weil die Mehrheit der Bevölkerung für die Fusionen gestimmt hätte. Letztlich hatten bis

5 Darunter fielen erstens eine Demokratiereform, die die Abschaffung des Proporzes, eine Verkleinerung der Landesregierung ( 7 statt 9 Landesräten) sowie des Landtages (48 statt 56 Mandaten) und eine Kürzung der Parteienförderung um $15 \%$ vorsah. Zweiter Aspekt war eine umfassende Verwaltungsreform, mit einer Aufgaben- und Organisationsreform (wie der Gemeindestrukturreform und Bezirksreform), einem flexiblen Personalmanagement und einer Haushaltsreform. Der dritte Pfeiler beinhaltete eine Spitalsreform, die dem „Regionalen Strukturplan Gesundheit Steiermark 2020“ mit Schwerpunkt-Spitälern folgte.

6 Unter den BürgermeisterInnen ging zu Beginn der Vorbereitungen der Gemeindestrukturreform die Furcht vor einem kommunalen Kahlschlag wie Ende der I960er um, wo die überwiegende Mehrzahl an Zusammenlegungen per Gesetz durchgesetzt wurde.

7 Im Jänner 2013 wurde die Gemeindeinitiative auch als Verhandlungspartner vom Land Steiermark anerkannt. Siehe dazu unter anderem http://forum.stlambrecht.at/gemeindeinitiative/GI_teiln_ gden.pdf. Nach dem II.Dezember 2012 sind zu den 107 Gemeinden noch zahlreiche hinzugekommen, der Höchststand war mit I 40 beziffert. I2I Gemeinden wurden aber am öftesten genannt, wodurch auch diese $\mathrm{Zahl}$ verwendet wurde.

8 Sofern zumindest 80 Gemeinden innerhalb von 6 Wochen einen Gemeinderatsbeschluss dafür gefasst hätten. 
zum Stichtag 30.09.20I3 viele Gemeinden ihre Abwehrhaltung aufgegeben, sodass in 306 von 385 Gemeinden (79.5\%) auch Gemeinderatsbeschlüsse für eine Vereinigung gefasst werden konnten (Wlattnig 20I4, 250-252). ${ }^{9}$ Mit OI.OI.2015 wurde dann die zweite vom Land verordnete Gemeindestrukturreform abgeschlossen.

\section{Empirisches Modell und Datendeskription}

Die Entwicklung der Ergebnisse in den Gemeinden wird anhand des Difference-in-Difference-Ansatzes überprüft. Diese Methode wird angewandt um die reine Veränderung durch die Zäsur Gemeindestrukturreform erfassen zu können. Hierbei werden die Veränderungen einer Treatmentgruppe (fusionierte Gemeinden) den Veränderungen einer Kontrollgruppe (nicht fusionierte Gemeinden) gegenübergestellt. Der Zeitpunkt des Eingriffs unterteilt die Gemeinden in eine Phase vor und nach dem Treatment. Die Trends werden somit auf Basis einer zeitlichen Veränderung verglichen. Damit sollte sich der kausale Effekt ergeben, der dem Faktor „Zusammenlegung" geschuldet ist.

Zentrale Annahme ist, dass die Trends in den Gemeinden ohne das Treatment Fusion die gleichen gewesen wären. Zwischen den Gemeinden kann es Unterschiede geben, diese werden anhand der Fixed-Effects für die Gemeinden einbezogen. Die Annahme gleicher Trends kann auch über mehrere Perioden überprüft werden (Vgl. Angrist/Pischke 2009, 227-230). Die Differencein-Difference-Schätzung wird robuster, wenn Daten vor dem Treatment einfließen um einen deutlichen Trend aufzuzeigen, der in die Post-Treatment-Phase hochgerechnet wird. Der längerfristige Trend wurde in diesem Fall mit den vier Wahlen vor dem Treatment bestimmt. Der Ansatz funktioniert in der Berechnung ganz allgemein für Gruppen, die von einer Policy-Maßnahme betroffen sind oder nicht (Vgl. Angrist/Pischke 2009, 237240). Hinzugesagt werden muss, dass die entsprechende Anzahl an Stimmen pro Partei jeweils auf Basis der neuen Gemeindegrenzen bestimmt wurden. ${ }^{10}$

Die Daten umfassen die Ergebnisse von Nationalrats-, Landtags- und Gemeinderatswahlen. Der bearbeitete Datensatz beinhaltet drei unterschiedlich lange Untersuchungszeiträume. Bei den Nationalratswahlen wurden die fünf Wahlgänge ab 1999 (1999, 2002, 2006, 2008, 2013) inkludiert, bei den Landtags- und Gemeinderatswahlen jeweils die fünf Urnengänge seit 1995(1995, 2000, 2005, 2010, 2015). Primär werden die letzten bei-

9 Das Steiermärkische Gemeindestrukturreformgesetz (StGsrG)wurde mit den Stimmen der Regierung aus SPÖ und ÖVP am 17.12.2013 im Landtag beschlossen und am 02.04.20I4 kundgemacht.

Io Hier ist die bereits genannte Studie von Heinz P. Wassermann und seinem Team (2016) anzuführen, die in ähnlicher Art und Weise vorgehen und dabei von einem "fiktiven" Vergleich sprechen. den Wahlen betrachtet, wenngleich auch der langfristige Vergleich interessante Aspekte herausstreicht. Einige kleinere Korrekturen waren wegen Grenzverschiebungen zwischen Bezirken, Gemeindeaufteilungen und Nachwahlen nötig. Wichtig ist anzumerken, dass die Landeshauptstadt Graz ausgeschlossen wurde, da diese einem anderen Wahlzyklus unterliegt.

Die erste Regressionsgleichung wird im Basismodell herangezogen - der Unterscheidung zwischen Fusion und Nicht-Fusion. Die zweite und dritte Regressionsgleichung verwenden eine feinere Aufgliederung der Gemeinden, nach jenen Gemeinden die geklagt oder sich in der Gemeindeinitiative engagiert haben. Die zu erklärenden Variablen sind die Wahlergebnisse der einzelnen Parteien - in absoluten Stimmen gemessen.
(I) PARTEI $_{2015 / 2013}=\alpha+\beta_{0}{ }^{*} \mathrm{t}+\beta_{1}{ }^{*}$ FUSION $+\beta_{2}{ }^{*}\left(\mathrm{t}^{*}\right.$ FUSION $)+\varepsilon_{1}$
(2) PARTEI $_{201 / 2013}=\alpha+\beta_{0}{ }^{*} \mathrm{t}+\beta_{1}{ }^{*}$ KLAGE $+\beta_{2}{ }^{*}\left(\mathrm{t}^{*}\right.$ KLAGE $)+\varepsilon_{\mathrm{i}}$
(3) PARTEI ${ }_{2015 / 2013}=\alpha+\beta_{0}{ }^{*} t+\beta_{1}{ }^{*}$ INITIATIVE $+\beta_{2}{ }^{*}\left(t^{*}\right.$ INITIATIVE $)+\varepsilon_{\mathrm{i}}$

Die folgenden Variablen sind Dummys: Die Variable FUSION beinhaltet sämtliche Gemeinden, die 2013/2015 fusioniert wurden. Die Variable KLAGE umfasst all jene fusionierten Gemeinden, die sich mit ihrer Klage gegen die Fusion ihrer Gemeinde an den Verfassungsgerichtshof gewandt haben. KLAGE wurden 29 Gemeinden zugeordnet, wobei es in einigen neu zusammengesetzten Gemeinden mehrere Klagen der alten Gemeinden gab. INITIATIVE ist eine Variable für alle Gemeinden, wo gegen die Fusionspläne bereits vor der Nationalratswahl 2013 mobilisiert wurde und es von einigen BürgermeisterInnen einen Wahlvorschlag gegen ihre eigenen Parteien gegeben hat. Diese Gruppe umfasst 89 Gemeinden. Die Zugehörigkeit einer Gemeinde zu KLAGE ist damit nicht ausgeschlossen, ebenso nicht, dass die entsprechende Gemeinde gar nicht fusioniert worden ist.

\section{Ergebnisse}

\subsection{Nationalratswahl 2013}

Bei der Nationalratswahl am 29.September 2013 haben ÖVP wie SPÖ deutliche Verluste sowohl in Fusions- als auch Nicht-Fusionsgemeinden hinnehmen müssen, während die FPÖ überall stark zulegt. In der Regression zum Basismodell (Gleichung I) ist aber nur das ÖVPErgebnis signifikant, die SPÖ-Verluste sind nicht signifikant. Die ÖVP verliert um 20\% mehr an Stimmen in zusammengelegten Gemeinden. Die FPÖ-Zugewinne sind ebenso signifikant stärker in Fusionsgemeinden ( $\mathrm{p}$-Wert $<\mathrm{O}, \mathrm{O} 0 \mathrm{O})$, die Ergebnisse von KPÖ und Grüne sind insignifikant. Bei allen Wahlen ist nicht nur der 
Vergleich unmittelbar vor und nach dem Treatment ${ }^{\mathrm{II}}$ interessant, sondern auch der langfristige Trend. Für den Langfristvergleich fließen die letzten fünf Wahlen ein. Nimmt man die Periode von 1999 bis 2013 als Untersuchungszeitraum verändert sich die Signifikanz der ÖVPund FPÖ-Ergebnisse nicht. Auffällig ist, dass die ÖVPVerluste stärker werden und höchst signifikant bleiben. Die FPÖ gewinnt dagegen in der langen Frist nur mehr ein Drittel so stark hinzu und der Zuwachs ist auch nur mehr schwach signifikant. Die Zugewinne der KPÖ und Grünen wie auch die Verluste der SPÖ verändern sich nur geringfügig und bleiben insignifikant.

In diesem Zusammenhang stellt sich die Frage, ob sich klagende Gemeinden anders verhalten haben - im Kontrast zu sämtlichen anderen Gemeinden (Gleichung 2). Die ÖVP- und SPÖ-Verluste sowie die FPÖ-Zugewinne sind in diesem Vergleich recht solide, aber keineswegs signifikant. Als weiterer Aspekt wurde der Einfluss der Gemeindeinitiative überprüft (Gleichung 3). Die ÖVPVerluste und FPÖ-Zugewinne schwächen sich nochmals ab, die SPÖ verliert nur mehr marginal. Die Ergebnisse sind aber wiederum insignifikant.

Zusammenfassend lässt sich bereits aus der Nationalratswahl ein Muster ableiten, welches sich bei den beiden anderen Wahlen wiederholt: Die ÖVP verliert signifikant stärker in Fusionsgemeinden, die FPÖ gewinnt signifikant stärker in Fusionsgemeinden. Die SPÖ verliert überall in etwa gleich stark, während die KPÖ und die Grünen kaum Unterschiede aufweisen und je nach Dimension entweder leichte Zugewinne oder leichte Verluste hinnehmen mussten. Die Ergebnisse bleiben auch im längerfristigen Trend im Wesentlichen identisch. Eine weitere Unterscheidung der Fusionsgemeinden liefert hier kaum zusätzlichen Erklärungsgehalt. Bei der Nationalratswahl ist der Boykottaufruf trotz der gegebenen medialen Präsenz keine wesentliche Erklärung für die starken SPÖ- und ÖVP-Verluste.

\subsection{Gemeinderatswahlen 2015}

Bei den Gemeinderatswahlen am 22.März 2015 sind die Verluste der Reformpartner vergleichsweise überschaubar ausgefallen. Dies äußert sich auch in den Regressionsanalysen, beim Vergleich von Fusions- zu NichtFusionsgemeinden wiederholt sich das Schema der Nationalratswahl eineinhalb Jahre zuvor. Die ÖVP verliert insgesamt fast gleich stark wie die SPÖ, allerdings sind die ÖVP-Verluste signifikant, da sie dreimal stärkere Verluste in zusammengelegten Gemeinden aufweisen (um 225\% höher als in nicht-fusionierten Gemeinde). Bei der SPÖ verlaufen die Einbußen in beiden Kategorien wie zuvor recht parallel. Die FPÖ gewinnt zwar recht

II Im Falle der Nationalratswahl noch knapp vor der Finalisierung, aber schon sehr weit fortgeschritten. stark hinzu, allerdings war sie 2015 fast in jeder Gemeinde präsent, nicht wie noch 20 Io nur in knapp der Hälfte. Deshalb ist der um zwei Drittel stärkere Zugewinn in fusionierten Gemeinden fast zwangsläufig, dennoch ist dieser unter Berücksichtigung größerer Präsenz noch hoch signifikant. Die KPÖ gewinnt ebenfalls substantiell hinzu, die Grünen leicht, beide Parteien allerdings ohne wesentlichen Unterschied zwischen den Gemeindetypen. Das Ergebnis der sonstigen Listen ist hoch signifikant, diese haben also in Fusionsgemeinden besonders stark reüssiert. Dort weisen sie dreimal so starke Zugewinne wie in nicht betroffenen Gemeinden auf.

Auf Basis des längerfristigen Trends zwischen den Gemeinderatswahlen von 1995 bis 2015 fallen die ÖVPVerluste um einiges geringer aus, allerdings immer noch mit signifikanten Unterschieden zwischen Fusions- und Nicht-Fusionsgemeinden. Bei der SPÖ sind die Verluste in beiden Untersuchungszeiträumen nahezu identisch und wiederum insignifikant. Langfristig verliert die SPÖ dennoch deutlich stärker gegenüber der ÖVP. Auch die FPÖ-Zugewinnen relativieren sich in der langen Frist etwas. In den I990ern hatte die Partei schon weit mehr Ortsparteien, dennoch sind die Differenzen zwischen den Gemeindetypen trotz dieser Tatsache hoch signifikant. Bei den Grünen fällt der kleine Zuwachs noch einmal geringer aus, bei der KPÖ hingegen sind die Zuwächse enorm. Beide Ergebnisse bleiben aber insignifikant. Die KPÖ-Zugewinne sind auch damit erklärbar, dass die Partei langsam mehr Listen aufstellt. Die Sonstigen Listen gewinnen zwar auch langfristig am meisten hinzu, allerdings sind die Unterschiede nicht mehr signifikant. Parteiunabhängige Listen konnten schon früher - 1995 und 2010 - teilweise starke Ergebnisse erreichen, wurden aber 2000 und 2005 deutlich zurückgedrängt wurden. Die Zahl der antretenden Bürgerlisten unterliegt starken Schwankungen über die Zeit. Die Formierung sonstiger Listen war 2015 wesentlich von den landespolitischen Begleitumständen beeinflusst worden. Bei Bürgerlisten scheint bei der Gemeinderatswahl 2015 tatsächlich ein Fusionseffekt zum Tragen gekommen zu sein.

Bei den Gleichungen 2 und 3 geht die Signifikanz bis auf ein Ergebnis vollständig verloren. Erwähnenswert sind bei klagenden Gemeinden die deutlich stärkeren SPÖ-Verluste und die weit geringeren FPÖ-Zugewinne. Gerade in diesen Gemeinden fuhren die sonstigen Listen ihre größten Erfolge ein. Ihr Stimmenzuwachs in Fusionsgemeinden (gegenüber Nicht-Fusionsgemeinden) ist der höchste gemessene signifikante Wert. Die Gemeindeinitiative hatte keine signifikanten Unterschiede zwischen den Gemeinden zur Folge, sämtliche Gewinne und Verluste wurden geringer.

Insgesamt können klare Parallelen zur Nationalratswahl 2013 hergestellt werden. Die ÖVP-Verluste sind wie die FPÖ-Zugewinne signifikant in der Unterscheidung zwischen fusionierten und nicht-fusionierten Gemein- 
Abbildung 1: Difference-in-Difference-Regression der 3 großen Parteien (Nationalratswahl 2008 vs. 2013, Kategorie FUSION)

\begin{tabular}{|c|c|c|c|}
\hline & ÖVP & SPÖ & FPÖ \\
\hline \multirow[t]{2}{*}{ treated } & $492.673^{\star * *}$ & $468.498^{\star * \star}$ & $121.961^{* * *}$ \\
\hline & $p=0.000$ & $p=0.000$ & $p=0.0004$ \\
\hline \multirow[t]{2}{*}{ time } & $-106.763^{\star \star *}$ & $-129.546^{*}$ & $199.061^{\star \star *}$ \\
\hline & $p=0.006$ & $p=0.079$ & $p=0.000$ \\
\hline \multirow[t]{2}{*}{ did } & $-184.904^{* * *}$ & -171.090 & $220.552^{\star * *}$ \\
\hline & $p=0.002$ & $p=0.119$ & $p=0.00001$ \\
\hline \multirow[t]{2}{*}{ Constant } & $469.994^{\star * *}$ & $494.424^{\star \star \star}$ & $132.241^{\star * *}$ \\
\hline & $p=0.000$ & $p=0.000$ & $p=0.000$ \\
\hline Observations & 572 & 572 & 572 \\
\hline $\mathrm{R}^{2}$ & 0.308 & 0.104 & 0.325 \\
\hline Adjusted $\mathrm{R}^{2}$ & 0.305 & 0.099 & 0.321 \\
\hline Residual Std. Error $(\mathrm{df}=568)$ & 338.711 & 651.270 & 284.811 \\
\hline F Statistic $(\mathrm{df}=3 ; 568)$ & $84.343^{\star \star *}$ & $21.905^{\star \star *}$ & $91.011^{\star \star *}$ \\
\hline
\end{tabular}

Abbildung 3: Difference-in-Difference-Regression der 3 großen Parteien (Gemeinderatswahlen 2010 vs. 2015, Kategorie FUSION)

\begin{tabular}{lccc}
\hline & ÖVP & SPÖ & FPÖ \\
\hline treated & $695.876^{* * *}$ & $539.860^{* * *}$ & $87.658^{* *}$ \\
& $\mathrm{p}=0.000$ & $\mathrm{p}=0.00000$ & $\mathrm{p}=0.022$ \\
time & -43.705 & -87.939 & $73.257^{*}$ \\
& $\mathrm{p}=0.479$ & $\mathrm{p}=0.348$ & $\mathrm{p}=0.053$ \\
did & $-170.764^{*}$ & -159.836 & $116.635^{* *}$ \\
& $\mathrm{p}=0.062$ & $\mathrm{p}=0.251$ & $\mathrm{p}=0.026$ \\
Constant & $670.609^{* * *}$ & $554.747^{* * *}$ & $150.450^{* * *}$ \\
& $\mathrm{p}=0.000$ & $\mathrm{p}=0.000$ & $\mathrm{p}=0.00000$ \\
Observations & 574 & 567 & 454 \\
$\mathrm{R}^{2}$ & 0.251 & 0.082 & 0.126 \\
Adjusted $\mathrm{R}^{2}$ & 0.247 & 0.077 & 0.120 \\
Residual Std. Error & $543.973(\mathrm{df}=570)$ & $824.029(\mathrm{df}=563)$ & $276.478(\mathrm{df}=450)$ \\
F Statistic & $63.512^{* * *}(\mathrm{df}=3 ; 570)$ & $16.748^{* * *}(\mathrm{df}=3 ; 563)$ & $21.628^{* * *}(\mathrm{df}=3 ; 450)$ \\
\hline Note: ${ }^{*} \mathrm{p}<0.1 ; * * \mathrm{k}<0.05 ; * * * 00$ & &
\end{tabular}

den. Die SPÖ-Verluste sind wiederum insignifikant, ebenso die Zugewinne der KPÖ wie der Grünen. In den weiteren Analysedimensionen werden sämtliche Ergebnisse insignifikant. Hervorzuheben ist das Ergebnis der sonstigen Listen. Wie bereits aus der deskriptiven Analyse zu erwarten, sind Bürger- und Namenslisten neben den Freiheitlichen die großen Gewinner der Gemeinderatswahlen. In allen Varianten sind die Effekte deutlich positiv, aber nicht immer signifikant. Die primäre Unterscheidung von fusionierten zu nicht-fusionierten $\mathrm{Ge}-$ meinden ist im Vergleich $2015 \mathrm{zu} 2010$ hoch signifikant. Im längerfristigen Vergleich - ab 1995 gemessen - sind die Zugewinne nicht mehr signifikant. Aus jetziger Sicht ist von einem Einmaleffekt auszugehen, wenngleich eine Persistenz dieses Fusionseffektes möglich ist. Die Gemeindestrukturreform ist also mancherorts ein wesent-
Abbildung 2: Difference-in-Difference-Regression der 3 großen Parteien (Nationalratswahl 2008 vs. 2013, Kategorie KLAGE)

\begin{tabular}{|c|c|c|c|}
\hline & ÖVP & SPÖ & FPÖ \\
\hline \multirow[t]{2}{*}{ treated } & $279.858^{* \star \star}$ & $538.060^{\star \star \star}$ & $265.535^{\star \star *}$ \\
\hline & $p=0.00001$ & $p=0.00003$ & $p=0.0004$ \\
\hline \multirow[t]{2}{*}{ time } & $-122.054^{\star \star \star}$ & $-145.543^{\star \star \star}$ & $106.058^{\star \star *}$ \\
\hline & $p=0.00001$ & $p=0.010$ & $p=0.002$ \\
\hline \multirow[t]{2}{*}{ did } & -127.589 & -148.315 & 103.692 \\
\hline & $p=0.132$ & $p=0.407$ & $p=0.322$ \\
\hline \multirow[t]{2}{*}{ Constant } & $504.035^{\star \star \star}$ & $564.190^{* \star \star}$ & $333.787^{\star * *}$ \\
\hline & $p=0.000$ & $p=0.000$ & $p=0.000$ \\
\hline Observations & 572 & 572 & 572 \\
\hline $\mathrm{R}^{2}$ & 0.091 & 0.061 & 0.084 \\
\hline Adjusted $\mathrm{R}^{2}$ & 0.087 & 0.056 & 0.079 \\
\hline Residual Std. Error $(\mathrm{df}=568)$ & 300.461 & 634.885 & 371.277 \\
\hline F Statistic $(d f=3 ; 568)$ & $19.025^{\star \star *}$ & $12.250^{\star \star *}$ & $17.303^{\star * *}$ \\
\hline
\end{tabular}

Note: ${ }^{*} \mathrm{p}<0.1 ;{ }^{* *} \mathrm{p}<0.05 ;{ }^{* * *} \mathrm{p}<0.01$

Abbildung 4: Difference-in-Difference-Regression der 3 kleinen Parteien (Gemeinderatswahlen 2010 vs. 2015, Kategorie KLAGE)

\begin{tabular}{lccc}
\hline & KPÖ & GRÜNE & SONSTIGE \\
\hline treated & 72.125 & 26.971 & -47.553 \\
& $\mathrm{p}=0.734$ & $\mathrm{p}=0.539$ & $\mathrm{p}=0.660$ \\
time & -13.917 & $53.385^{* *}$ & 35.232 \\
& $\mathrm{p}=0.890$ & $\mathrm{p}=0.022$ & $\mathrm{p}=0.621$ \\
did & -3.483 & 1.580 & $309.501^{*}$ \\
& $\mathrm{p}=0.990$ & $\mathrm{p}=0.979$ & $\mathrm{p}=0.060$ \\
Constant & $306.875^{* * *}$ & $162.938^{* * *}$ & $368.753^{* * *}$ \\
& $\mathrm{p}=0.0001$ & $\mathrm{p}=0.000$ & $\mathrm{p}=0.000$ \\
Observations & 56 & 163 & 182 \\
$\mathrm{R}^{2}$ & 0.006 & 0.046 & 0.037 \\
Adjusted $\mathrm{R}^{2}$ & -0.052 & 0.028 & 0.021 \\
Residual Std. Error & $344.035(\mathrm{df}=52)$ & $134.065(\mathrm{df}=159)$ & $429.869(\mathrm{df}=178)$ \\
F Statistic & $0.098(\mathrm{df}=3 ; 52)$ & $2.578^{*}(\mathrm{df}=3 ; 159)$ & $2.274^{*}(\mathrm{df}=3 ; 178)$ \\
\hline Note: ${ }^{*} \mathrm{p}<0.1 ; *{ }^{* *}<0.05 ; * *{ }^{* *}<0.01$ & &
\end{tabular}

liches Thema gewesen. Vor allem aber sind die Fusionen bei Gemeinden, die mit ihrem Widerstand bis vor den Verfassungsgerichtshof gingen, eine gute Erklärung. Sonstige Listen liegen in diesen Gemeinden signifikant besser als in sämtlichen anderen Gemeinden. Somit lässt sich sagen, dass sich die Muster der Nationalratswahl fortsetzen, wobei die Ergebnisse der SONSTIGEN die wesentliche Erkenntnis darstellen.

\subsection{Landtagswahl 2015}

Die Landtagswahl am 3I.Mai 2015 endet für die Reformpartner mit massiven Verlusten, die zu großen Teilen von den Freiheitlichen aufgenommen wurden. Bei der Regression mit dem Treatment Fusionen zeigen sich die bekannten Muster wieder. Einzig sind nun die Verluste 
der ÖVP wie auch die Gewinne der FPÖ höchst signifikant (p-Werte <o,ooo), die SPÖ-Verluste insignifikant, KPÖ und Grüne legen nur insignifikant zu. In der längerfristigen Perspektive gibt es noch ein weiteres signifikantes Ergebnis. Die SPÖ verliert im Zeitraum 1995 bis 2015 signifikant stärker in Fusionsgemeinden. Dies ist in der Analyse der einzig signifikante Wert für die Sozialdemokraten. Die ÖVP-Verluste sind langfristig wie die FPÖ-Zugewinne höchst signifikant. Während die FPÖGewinne etwas geringer ausfallen und den SPÖ-Verlusten in etwa entsprechen, verliert die ÖVP langfristig sogar dramatisch mehr. Die Wahlbeteiligung ist insofern ein Faktor, da sie auf Landesebene am schnellsten gefallen ist und deshalb die Parteien relativ am wenigsten zulegen oder einbüßen.

In den weiteren Abstufungen zeigen sich diesmal auch signifikante Ergebnisse. In klagenden Gemeinden verliert die ÖVP übermäßig stark und signifikant. Die SPÖ verliert zwar auch mehr an Stimmen, aber nicht signifikant mehr. Die FPÖ kann in diesen Gemeinden weit stärker zulegen und dies höchst signifikant. Auch die Grünen legen hier deutlich stärker zu, aber ohne signifikante Divergenz. In den „initiativen“ Gemeinden sind die ÖVP-Verluste wiederum signifikant und übersteigen die ebenfalls signifikanten FPÖ-Zugewinne. Bei der SPÖ fallen die Verluste in dieser Dimension weniger drastisch aus. Das Ergebnis ist dadurch insignifikant. Die Nicht-Signifikanz dieses Ergebnisses verdeutlicht, dass die SPÖ ihre Verluste in allen Gruppen von Gemeinden nahezu gleichmäßig eingefahren hat.

Gesamt gesehen weichen die Ergebnisse bei der Landtagswahl teilweise deutlich von den vorangegangenen Nationalrats- und Gemeinderatswahlen ab. Die Richtung der Effekte bleibt zwar bei allen Wahlen für alle Parteien gleich, auffällig ist bei der Landtagswahl aber, dass weit mehr Dimensionen signifikante Effekte aufweisen. Die ÖVP verliert in den Fusionsgemeinden signifikant mehr als in nicht-fusionierten Gemeinden, sowohl in der kurzen als auch der langen Frist. Gleiches gilt für die FPÖ vice versa. Allerdings sind bei diesen beiden Parteien nun auch die Kategorien KLAGE und INITIATIVE signifikant. Die ÖVP verliert in klagenden wie auch in initiativen Gemeinden signifikant mehr als in den restlichen Gemeinden, die FPÖ-Zugewinne sind in diesen beiden Kategorien jeweils höchst signifikant. Die Veränderungen bei der KPÖ und den Grünen sind wie bei den beiden anderen Wahlen in allen Fällen nicht signifikant. Interessant ist, dass es bei dieser Wahl auch bei der SPÖ ein signifikantes Ergebnis zu beobachten gibt. Zwar verliert die SPÖ bei allen Wahlen in allen Kategorien recht parallel, dafür ist nun das Ergebnis für die Unterscheidung von fusionierten zu nicht-fusionierten Gemeinden in der langen Frist signifikant. ${ }^{12}$

I2 Die fusionierten Gemeinden waren tendenziell ländliche Kleinge-
Der Frust über die Reformen der Landesregierung wurde so gesehen mehrheitlich auf die Landtagswahlen übertragen. Dahingehende Vermutungen waren zwar im Vorfeld von vielen politischen Beobachtern so geäußert worden, die etwas geringeren Verluste bei den Gemeinderatswahlen ließen wohl manche SPÖ- oder ÖVPPolitiker zu früh aufatmen. Umfragen kurz vor der Wahl legten zwar klare Verluste für die Reformpartner nahe, das FPÖ-Ergebnis wurde aber nicht ansatzweise prognostiziert (Wassermann 2016). ${ }^{\mathrm{I3}}$

\subsection{Zusammenfassung}

Insgesamt sollten sämtliche Ergebnisse der Parteien einzeln für sich analysiert werden, auch wenn sich interessante Muster bei sämtlichen Wahlen durchsetzen. Die Fusionen haben in Teilen das Ergebnis mitbeeinflusst, aber nicht in dem Ausmaß wie es die phasenweise große mediale Präsenz dieses Themas vermuten ließ. Die Gemeindestrukturreform war eben nur ein Teilaspekt eines der drei Pfeiler der Reformpartnerschaft.

Insbesondere die hohen Verluste der SPÖ sind kaum durch die Gemeindestrukturreform zu erklären. Einzig signifikantes Ergebnis ist für die Landtagswahl in der langfristigen Perspektive. Dabei wurde die Aufbauarbeit von Franz Voves, wodurch erstmals der Landeshauptmannsessel von der ÖVP übernommen wurde, revidiert. Durch die letzten Ergebnisse wurde die steirische SPÖ wieder stärker auf ihre Stammwählerschaft zurückgestutzt, wie es Anfang und Mitte der I990er schon der Fall war (Marko 1992). Die Gemeinderatswahlen waren zudem eine Prolongierung der Verluste von 20I0. Die starke Personalisierung der Landespolitik ist in diesem Zusammenhang auch ein guter Erklärungsgrund für die teils großen, nicht alltäglichen Wählerwanderungen zwischen SPÖ und ÖVP. Bei der Bewertung der SPÖ-Verluste müssen den sozialen Einschnitten und den strukturellen Problemen der Partei in Landgemeinden mehr Gewicht zugeordnet werden.

Die ÖVP hat durch die Gemeindestrukturreform stärkere zusätzliche Verluste eingefahren als dies beim Koalitionspartner SPÖ der Fall war. Als konservative Partei wird sie als Garant für den Erhalt von Strukturen gesehen. Von daher stand für die Volkspartei mehr auf dem Spiel. Die Ergebnisse verdeutlichen, dass die ÖVP bei allen drei Wahlen in Fusionsgemeinden signifikant stärker verloren hat. Die Verluste sind zwar damit nicht per se erklärt, aber die ungleich stärkeren Verluste in

meinden, in denen die Sozialdemokraten traditionell schwächer aufgestellt sind. In der Analyse zeigte sich, dass die Erringung des Landeshauptmannsessels aber ohne temporäre Zugewinne in solchen Gemeinden nicht möglich gewesen wäre.

I3 Beispielhaft sei hier der Durchschnitt der letzten 8 Umfragen der Politik-Homepage neuwal.at angeführt: Für die SPÖ wurden 31.6\% ausgewiesen; für die ÖVP 29.1\%; für die FPÖ 20.5\%; für die GRÜNEN $7.8 \%$ und für die KPÖ $4.6 \%$. 
Abbildung 5: Difference-in-Difference-Regression der 3 großen Parteien (Landtagswahl 2010 vs. 2015, Kategorie FUSION)

\begin{tabular}{|c|c|c|c|}
\hline & ÖVP & SPÖ & FPÖ \\
\hline \multirow[t]{2}{*}{ treated } & $492.673^{\star \star *}$ & $468.498^{\star \star \star}$ & $121.961^{\star \star *}$ \\
\hline & $p=0.000$ & $p=0.000$ & $p=0.0004$ \\
\hline \multirow[t]{2}{*}{ time } & $-106.763^{* \star *}$ & $-129.546^{*}$ & $-199.061^{\star \star *}$ \\
\hline & $p=0.006$ & $p=0.079$ & $p=0.000$ \\
\hline \multirow[t]{2}{*}{ did } & $-184.904^{\star \star *}$ & -171.090 & 220.552 \\
\hline & $p=0.002$ & $p=0.119$ & $p=0.00001$ \\
\hline \multirow[t]{2}{*}{ Constant } & $469.994^{\star \star \star}$ & $494.424^{\star \star \star *}$ & $132.241^{* * *}$ \\
\hline & $p=0.000$ & $p=0.000$ & $p=0.000$ \\
\hline Observations & 572 & 572 & 572 \\
\hline $\mathrm{R}^{2}$ & 0.308 & 0.104 & 0.325 \\
\hline Adjusted $\mathrm{R}^{2}$ & 0.305 & 0.099 & 0.321 \\
\hline Residual Std. Error $(\mathrm{df}=568)$ & 338.711 & 651.270 & 284.811 \\
\hline F Statistic $(\mathrm{df}=3 ; 568)$ & $84.343^{\star \star *}$ & $21.905^{\star \star *}$ & $91.011^{\star \star \star}$ \\
\hline
\end{tabular}

Abbildung 6: Difference-in-Difference-Regression der 3 großen Parteien (Landtagswahl 2010 vs. 2015, Kategorie KLAGE)

\begin{tabular}{|c|c|c|c|}
\hline & ÖVP & SPÖ & FPÖ \\
\hline \multirow[t]{2}{*}{ treated } & $381.941^{* * *}$ & $566.298^{* \star *}$ & $152.663^{* * *}$ \\
\hline & $p=0.00000$ & $p=0.00002$ & $p=0.010$ \\
\hline \multirow[t]{2}{*}{ time } & $-170.770^{\star \star \star}$ & $-189.471^{\star \star \star}$ & $272.062^{\star * *}$ \\
\hline & $p=0.00000$ & $p=0.002$ & $p=0.000$ \\
\hline \multirow[t]{2}{*}{ did } & $-206.574^{*}$ & -194.564 & $253.110^{\star \star *}$ \\
\hline & $p=0.054$ & $p=0.291$ & $p=0.003$ \\
\hline \multirow[t]{2}{*}{ Constant } & $655.128^{\star \star *}$ & $650.047^{\star \star \star}$ & $172.233^{\star \star *}$ \\
\hline & $p=0.000$ & $p=0.000$ & $p=0.000$ \\
\hline Observations & 572 & 572 & 572 \\
\hline $\mathrm{R}^{2}$ & 0.105 & 0.068 & 0.258 \\
\hline Adjusted $\mathrm{R}^{2}$ & 0.100 & 0.063 & 0.254 \\
\hline Residual Std. Error $(\mathrm{df}=568)$ & 385.123 & 664.077 & 298.350 \\
\hline F Statistic (df = 3; 568) & $22.157^{\star * *}$ & $13.769^{\star \star \star *}$ & $65.810^{\star * *}$ \\
\hline
\end{tabular}

Tabelle 3: Signifikanztabelle der Regressionen

\begin{tabular}{|c|c|c|c|c|c|c|c|c|}
\hline Wahlen & Treatment & Zeitraum & ÖVP & SPÖ & FPÖ & КРÖ & GRÜNE & SONST \\
\hline \multirow[t]{4}{*}{ Nationalrat } & FUSION & $2008-2013$ & neg. *** & neg. & pos. ${ }^{* * *}$ & pos. & pos. & \\
\hline & & $1999-2013$ & neg. *** & neg. & pos. $* * *$ & pos. & pos. & \\
\hline & KLAGE & $2008-2013$ & neg. & neg. & pos. & pos. & pos. & \\
\hline & INITIATIVE & $2008-2013$ & neg. & neg. & pos. & pos. & pos. & \\
\hline \multirow[t]{4}{*}{ Gemeinderat } & FUSION & $2010-2015$ & neg. * & neg. & pos. ** & pos. & pos. & pos. ** \\
\hline & & $1995-2015$ & neg. * & neg. & pos. ** & pos. & pos. & pos. \\
\hline & KLAGE & $2010-2015$ & neg. & neg. & pos. & neg. & pos. & pos. * \\
\hline & INITIATIVE & $2010-2015$ & neg. & neg. & pos. & neg. & pos. & pos. \\
\hline \multirow[t]{4}{*}{ Landtag } & FUSION & $2010-2015$ & neg. *** & neg. & pos. *** & pos. & pos. & \\
\hline & & $1995-2015$ & neg. *** & neg. * & pos. $* * *$ & pos. & pos. & \\
\hline & KLAGE & $2010-2015$ & neg. * & neg. & pos. *** & pos. & pos. & \\
\hline & INITIATIVE & 2010-2015 & neg. * & neg. & pos. *** & 0 & pos. & \\
\hline
\end{tabular}

Fusionsgemeinden können als gewisser Malus interpretiert werden. Speziell bei den Gemeinderatswahlen ist diese Schlussfolgerung zulässig, da die Verluste in nicht betroffenen Gemeinden in einem überschaubaren Rahmen geblieben sind.
Die hohen Zugewinne der FPÖ sind zu einem guten Teil mit den Fusionen erklärbar, greifen aber bei teils erdrutschartigen Zugewinnen insgesamt zu kurz. Die Ablehnung der Reformpartnerschaft an sich korreliert recht stark mit dem FPÖ-Ergebnis bei der Landtags- 
wahl. Die Stimmungslage ist im Umfeld der analysierten Wahlen für Protest recht günstig gewesen, dieses Potential hat aber fast ausschließlich die FPÖ abschöpfen können. Angesichts der historischen Ergebnisse sind die Zugewinne bei der Nationalrats- und den Gemeinderatswahlen aber zu relativieren. Das Ergebnis der Landtagswahl ist ob der massiven Zugewinne überraschend ausgefallen, die Fusionen waren ein kleiner Bonus. Die FPÖ hat erst bei der Landtagswahl ihr Potential in hohem Maße erreichen können, weil es zum einen bei der Nationalratswahl ein starkes Team Stronach und bei den Gemeinderatswahlen überparteiliche Listen gab. Erst die Landtagswahlen waren die „wahren“ Wahlen über die Urheber der Reformen, wodurch hier die negative Stimmung vollständig zum Tragen kam.

Die Grünen und die KPÖ haben nur in Ansätzen von den Verlusten der Reformpartnerschaft profitiert. Gemeinden, die von der Gemeindestrukturreform betroffen waren, sind bei beiden Parteien unauffällig. Die sozialen Einschnitte der Reformpartnerschaft können für die leichten Zugewinne eher eine Erklärung liefern. Die Grünen haben insgesamt ihre in der Steiermark doch eher prekäre Lage etwas verbessern können, auch die KPÖ hat sich entgegen vieler Vermutungen im Landtag und einigen Bezirken etabliert. Dennoch können diese beiden Parteien nur in geringem Maß als Profiteure der Reformpartner-Verluste gelten. Hervorzuheben sind hingegen die sonstigen Listen. Bei den Gemeinderatswahlen haben Bürger- und Namenslisten in Fusionsgemeinden signifikant stärker gewonnen, aber nur gegenüber 2010 und nicht langfristig. Insbesondere in der Kategorie KLAGE gewinnen sie signifikant mehr. Bei nur einem Drittel an Gemeinden, wo überhaupt Listen angetreten sind, haben diese das Protestpotential gut ausgeschöpft. Die Proteste waren zwar nicht nur gegen Zusammenlegungen gerichtet, aber in Teilen sicher ein gewichtiger Faktor gewesen - vor allem in den klagenden Gemeinden.

\section{Conclusio und Diskussion}

Bei den Nationalratswahlen 2013 waren die Reformen nur teilweise umgesetzt und daher dem SPÖ- und ÖVPErgebnis noch nicht ganz so abträglich wie 2015, dennoch waren die steirischen Ergebnisse für die Bundesparteien der Reformpartner bereits auffällig schwach. Die Reformpartnerschaft stand zwar 2013 medial schon im Fokus, aber eher als Ausrede für die Bundespolitik. Die Diskussionen über die Fusionen und insbesondere der Boykottaufruf dürften überbewertet worden sein. Auch bei den Gemeinderatswahlen kam der Protest nur lokal zum Tragen und ließ damit nur eingeschränkte Rückschlüsse $\mathrm{zu}^{{ }^{14}}$ Verordnete Gemeindefusionen sind

I4 Solche rein lokalen Faktoren waren unter anderem der Kampf um riskant, allerdings bergen auch freiwillige Zusammenschlüsse - wie sie primär in der Schweiz erfolgen - keine Erfolgsgarantie in sich (Vgl. Steiner et al. 2014, I70). Die Motive und Interessenslagen sind unter Schweizer Gemeinden auch fast gänzlich andere (Vgl. Ladner et al. 2000, I53-I54).

Da die Steiermark als eines von zwei Bundesländern noch am indirekten Wahlsystem auf kommunaler Ebene festhält, sind die Gemeinderatswahlen primär Abstimmungen über die BürgermeisterInnen und weniger über Parteien oder Listen. Die Kommunalpolitik ist zwar nicht - wie oft unterstellt - unpolitisch und pragmatisch, wohl aber personenzentriert (Klammer 2000). VetospielerInnen, wie sie BürgermeisterInnen archetypisch darstellen, haben wesentlich zur Akzeptanz der Zusammenlegungen in der Bevölkerung beigetragen. Die Formierung von Widerstand über die Gemeindeinitiative oder das Einbringen von Verfassungsklagen ist von den Spitzen der Gemeinden initiiert worden. Die Feststellung eines Top-Down-Protests lässt dementsprechende Rückschlüsse auf die Wahlergebnisse zu. Zugleich formiert sich lokal überparteilicher Widerstand leichter über Listen, die anders als Ortsparteien nur horizontal - in der Gemeinde - verhaftet sind (Hämmerle 2000).

Die Landtagswahl war letztlich die Ebene, wo sich der Protest kumuliert hat, Fusionen bilden einen kleinen Teil davon ab. Insgesamt sind bestimmte (Arten von) Reformen eine riskante Angelegenheit für die politischen Machthaber. Die Erschütterung des Wählerstimmenmarktes durch große Reformen konnte in der Steiermark eindeutig beobachtet werden, insbesondere wo die Landespolitik zur Abstimmung stand. Der Reformgrad der einzelnen Maßnahmen aus wissenschaftlicher Sicht korreliert dennoch nicht immer mit dem subjektiven Empfinden der Bevölkerung. Lokal wurde speziell die Gemeindestrukturreform als Reform dritter Ordnung angesehen, obwohl diese aus objektiver Sicht - und auch aus Sicht der Mehrheit der steirischen Wahlbevölkerung - keinen derart gravierenden politischen Eingriff darstellte.

Die größere Distanz durch Fusionen - faktisch wie psychologisch - zieht eine Stärkung der Verwaltung gegenüber dem Gemeinderat nach sich. Stärkere Unzufriedenheit ist in diesem Sinne der ungenaueren Bedienung politischer Präferenzen geschuldet (Rösel 20I6b, 47). In Deutschland äußerte sich diese höhere Distanz in einem signifikanten und permanenten Rückgang der Wahlbeteiligung. In Baden-Württemberg halten diese Effekte beispielsweise seit den 1970ern an (Fritz/Feld 2015). Analog konnte Rösel (2016a) für die Steiermark diese unzureichende Repräsentation nachweisen, zudem existiert in Österreich eine allgemein stärkere Tendenz

den Erhalt der Expositur oder um eine Kleinschule, der Widerstand gegen ein Glashausprojekt oder ein Schubhaftzentrum oder einfach große Finanzprobleme der Gemeinde. 
zu Rechtspopulisten. Die hohen politischen Kosten von Gebietsreformen verdeutlichen zwei Statistiken: In der Steiermark hatte die einzelne Wählerstimme nach der Gemeindestrukturreform in der fusionierten Gemeinde um $80 \%$ weniger Gewicht als zuvor, zudem wurden Io\% der Fusionsgemeinden in Richtung "falscher" Mehrheiten $^{15}$ gedreht. Ein Viertel der WählerInnen in Fusionsgemeinden wurde demnach politisch schlechter gestellt (Rösel 20I6a, 2I). Diese hohen politischen Kosten konnten auch anhand unserer Ergebnisse nachgewiesen werden. Wie eingangs dargelegt ist das Risiko von Reformen groß, wenn die Verlierer viel offensichtlicher sind als die Gewinner. Insofern ist das „Abstrafen“ in der Wahlkabine Ausdruck gesteigerter Unzufriedenheit, da auch die Partizipationsmöglichkeiten schwinden. Im Allgemeinen kann man davon ausgehen, dass das Ausmaß an Einsparungen teilweise zu hinterfragen ist, während gleichzeitig substantielle politische Kosten entstehen.

Für die zukünftige Forschung stellt sich insbesondere die Frage nach der Persistenz dieser Effekte, ob beispielsweise eine dauerhaft niedrigere Wahlbeteiligung in fusionierten Gemeinden anhält. In diesem Zusammenhang ist die Frage nach den Wahlmotiven bei nachfolgenden Wahlen in der Steiermark von entsprechend großer Relevanz. Bei den darauffolgenden nationalen Wahlgängen wie der Bundespräsidentenwahl oder der Nationalratswahl waren andere Motive entscheidend. Im internationalen Vergleich, insbesondere gegenüber der Schweiz, aber auch den anderen Bundesländern, könnte zudem die Wirkung von Zwang oder Freiwilligkeit bei Fusionen im Detail eruiert werden. Offenheit der lokalen Politgrößen gegenüber mehr Kooperation oder einer Fusion scheint ein Faktor gewesen zu sein, da umgekehrt der Effekt von starkem Widerstand nachweislich groß war. Die Unterscheidungen zwischen Zwang und Freiwilligkeit sowie zwischen ökonomischer Rationalität (Betonung von Skaleneffekten) und dem sozialpsychologischen Wunsch nach der Beständigkeit von Strukturen könnten zudem intensiver behandelt werden.

\section{Literatur}

Acemoglu, Daron/James A. Robinson (200o), Political Losers as a Barrier to Economic Development, in: The American Economic Review, Vol. 90(2), I26-I3O.

Allers, Maarten A./J. Bieuwe Geertsema (2016), The effects of local government amalgamation on public spending, taxation, and service levels: Evidence from I5 years of municipal consolidation, in: Journal of $R e^{-}$ gional Science, Vol. 56(4), 659-682.

I5 Aus vormals SPÖ-Gemeinden wurden durch Zusammenlegungen ÖVP-Gemeinden und vice versa.
Angrist, Joshua D./Jörn-Steffen Pischke (2009), Mostly Harmless Econometrics: An Empiricists Companion, Princeton/Oxford: Princeton University Press

Blesse, Sebastian/Thushyanthan Baskaran (2016), Do Municipal Mergers Reduce Costs? Evidence from a German Federal State, in: Regional Science and Urban Economics, Vol. 59, 54-74.

Blom-Hansen, Jens/Kurt Houlberg/Søren Serritzlew (20I4), Size, Democracy, and the Economic Costs of Running the Political System, in: American Journal of Political Science, Vol. 58(4), 790-803.

Buti, Marco/Alessandro Turrini/Paul Van den Noord/Pietro Biroli/Mathias Thoenig/Ekaterina Zhuravskaya (2010), Reforms and re-elections in OECD countries, in: Economic Policy, Vol. 25(6I), 6I-II6.

Fernandez, Raquel/Dani Rodrik (199I), Resistance to Reform: Status Quo Bias in the Presence of IndividualSpecific Uncertainty, in: The American Economic Review, Vol. 8I(5), II46-II55.

Fritz, Benedikt/Lars P. Feld (2015), The Political Economy of Municipal Amalgamation: Evidence of Common Pool Effects and Local Public Debt, in: CESifo Working Paper, No. 5676

Garmann, Sebastian (2017), The effect of a reduction in the opening hours of polling stations on turnout, in: Public Choice, Vol. I7I(I-2), 99-II7.

Hansen, Sune Welling (2015), The Democratic Costs of Size: How Increasing Size Affects Citizen Satisfaction with Local Government, in: Political Studies, Vol. 63(2), 373-389.

Hämmerle, Walter (2000), Zwischen Konkurrenz und Konsens: Entscheidungsregeln im kommunalen politischen System, Opladen: Leske + Budrich

Klammer, Dieter (2000), Kommunalpolitiker und Ortsparteien in Österreich: Eine empirische Untersuchung der lokalen Positionseliten in Österreichs Gemeinden, Linz: Trauner

Ladner, Andreas/Daniel Arn/Ueli Friederich/Reto Steiner/ Jürg Wichtermann (2000), Gemeindereformen zwischen Handlungsfähigkeit und Legitimation, Bern: Universität Bern

Lassen, David D./Søren Serritzlew (2011a), Jurisdiction Size and Local Democracy: Evidence on Internal Political Efficacy from Large-scale Municipal Reform, in: American Political Science Review, Vol. IO5(2), 238258.

Lassen, David D./Søren Serritzlew (20Irb), Size and Equal Opportunity in the Democratic Process: The Effect of the Danish Local Government Reform on Inequality in Internal Political Efficacy, in: World Political Science Review, Vo. 7(I), I45-162.

Lehmbruch, Gerhard (1998), Parteienwettbewerb im Bundesstaat: Regelsysteme und Spannungslagen im Institutionengefüge der Bundesrepublik Deutschland, Opladen. Westdeutscher Verlag, 2.Auflage 
Lüchinger, Simon/Alois Stutzer (2002), Skalenerträge in der öffentlichen Kernverwaltung. Eine empirische Analyse anhand von Gemeindefusionen, in: Swiss Political Science Review, Vol. 8(I), 27-50.

Marko, Joseph (1992), Parteien und Wahlen in der Steiermark, in: Dachs, Herbert (Hg.), Parteien und Wahlen in Österreichs Bundesländern 1945-I99I, Wien/ München: Oldenbourg, 345-437.

Mayntz, Renate/Wolfgang Streeck (Hg.) (2003), Die Reformierbarkeit der Demokratie: Innovationen und Blockaden, Frankfurt/New York: Campus Verlag

Meyer, Christine Benedichte (2012), When Radical Reforms Are on the Agenda: Managing Politics in Government, in: The Journal of Applied Behavioral Science, Vol. 48(2), 194-224.

Moisio, Antti/ Roope Uusitalo (20I3), The Impact of $\mathrm{Mu}$ nicipal Mergers on Local Public Expenditures in Finland, in: Public Finance and Management, Vol. I3(3), I48-I66.

Pekari, Bernd (2016), Die mediale Resonanz auf die "Reformpartnerschaft" von SPÖ und ÖVP in der Steiermark, in: Karl, Beatrix/Wolfgang Mantl/Klaus Poier/ Manfred Prisching/Anita Ziegerhofer (Hg.), Steirisches Jahrbuch für Politik 2015, Wien-Köln-Weimar: Böhlau Verlag, 203-208.

Poier, Klaus (20I4), Nationalratswahl 20I3: Die österreichische Parteienlandschaft im Umbruch, in: Karl, Beatrix/Wolfgang Mantl/Klaus Poier/ Anita Prettenthaler-Ziegerhofer/Manfred Prisching/Bernd Schilcher (Hg.), Steirisches Jahrbuch für Politik 2013, Graz: Böhlau Verlag, I89-196.

Poier, Klaus (2015), 2005-2015 Das janusköpfige Dezennium, in: Politicum, Vol. 36(II8), 96-II3.

Poier, Klaus (2016), Die steirische Landtagswahl 2015: Erwartbares, Überraschungen, Lehren, in: Karl, Beatrix/Wolfgang Mantl/Klaus Poier/Manfred Prisching/ Anita Ziegerhofer (Hg.), Steirisches Jahrbuch für Politik 2015, Wien-Köln-Weimar: Böhlau Verlag, I37-I52.

Reingewertz, Yaniv (2012), Do Municipal Amalgamations Work? Evidence from Municipalities in Israel, in: Journal of Urban Economics, Vol. 72(2-3), 240-25I.

Rösel, Felix (2016), Die politischen Kosten von Gebietsreformen, in: ifo Dresden berichtet, Vol. 23(I), 2 I-25.

Rösel, Felix (2016), Sparen Gebietsreformen Geld? Ein Überblick über aktuelle Studien, in: ifo Dresden berichtet, Vol. 23(4), 45-49.

Schmidt, Manfred G. (2005), Politische Reformen und Demokratie: Befunde der vergleichenden Demokratie- und Staatstätigkeitsforschung, in: Vorländer, Hans (Hg.), Politische Reform in der Demokratie, Baden-Baden: Nomos, 45-62.

Steiner, Reto/Andreas Ladner/Pascal Reist, P. (20I4), Reformen in Kantonen und Gemeinden, Bern: Haupt Verlag

Steininger, Barbara (2004), Bürgermeister und Bürger- meisterinnen in Niederösterreich - kommunale Eliten im politischen System, in: Wagner, Manfred (Hg.), Niederösterreich: Menschen und Gegend, WienKöln-Weimar: Böhlau Verlag, 243-27I.

Tavares, Antonio F. (2018), Municipal amalgamations and their effects: a literature review, in: Miscellanea Geographica - Regional Studies on Development, Vol. 22(I), 5-15.

Tsebelis, George (1995), Decision Making in Political Systems: Veto Players in Presidentialism, Parliamentarism, Multicameralism and Multipartyism, in: British Journal of Political Science, Vol. 25(3), 289-325.

Tsebelis, George (1999), Veto Players and Law Production in Parliamentary Democracies: An Empirical Analysis, in: The American Political Science Review, Vol. 93(3), 59I-608.

Tsebelis, George (2000), Veto Players and Institutional Analysis, in: Governance, Vol. I3(4), 44I-474.

Vis, Barbara/Kees van Kersbergen (2007), Why and how do political actors pursue risky reforms, in: Journal of Theoretical Politics, Vol. I9(2), I53-I72.

Wassermann, Heinz P. (2016), Von weggebrochenen Hochburgen und zerbröckelnden Parteien, in: Karl, Beatrix/Wolfgang Mantl/Klaus Poier/Manfred Prisching/ Anita Ziegerhofer (Hg.), Steirisches Jahrbuch für Politik 2015, Wien-Köln-Weimar: Böhlau Verlag, I77$2 \mathrm{O} 2$.

Welan, Manfried (200o), Der Bürgermeister in Niederösterreich, Wien: Verlag Orac

Wlattnig, Wolfgang (2014), Streiflichter einer historischen Gemeindestrukturreform, in: Karl, Beatrix/ Mantl, Wolfgang/Poier, Klaus/Prettenthaler-Ziegerhofer, Anita/Prisching, Manfred/Schilcher, Bernd (Hg.), Steirisches Jahrbuch für Politik 2013, Graz: Böhlau Verlag, 247-252.

Wlattnig, Wolfgang/Manfred Kindermann (2016), 2015: Gemeindestrukturreform und Gemeinderatswahl in der Steiermark, in: Karl, Beatrix/Wolfgang Mantl/ Klaus Poier/Manfred Prisching/Anita Ziegerhofer (Hg.), Steirisches Jahrbuch für Politik 2015, Wien-KölnWeimar: Böhlau Verlag, I27-I36.

Yamada, Kyohei (2018), From a Majority to a Minority: How Municipal Mergers in Japan Changed the Distribution of Political Powers and the Allocation of Public Services Within a Merged Municipality, in: Urban Affairs Review, Vol. 54(3), 560-592.

\section{Autor}

Florian Teurezbacher ist wissenschaftlicher Mitarbeiter am Institut für International Business der IMC Fachhochschule Krems. Seine Forschungsschwerpunkte liegen im Bereich der Regionalforschung und der Arbeitsmarktökonomie. 